\title{
Identification of hub genes and small molecule therapeutic drugs related to breast cancer with comprehensive bioinformatics analysis
}

\author{
Mingqian Hao ${ }^{1}$, Wencong Liu ${ }^{\text {Corresp., }}{ }^{1}$, Chuanbo Ding ${ }^{1}$, Xiaojuan Peng ${ }^{1}$, Yue Zhang ${ }^{1}$, Huiying Chen ${ }^{1}$, Ling Dong ${ }^{1}$, \\ Xinglong Liu ${ }^{1}$, Yingchun Zhao ${ }^{1}$, Xueyan Chen ${ }^{1}$, Sadia Khatoon ${ }^{1}$, Yinan Zheng \\ ${ }^{1}$ School of Chinese Medicinal Materials, Jilin Agricultural University, Changchun, Jilin, China \\ Corresponding Author: Wencong Liu \\ Email address: xiaodiliqian@163.com
}

Breast cancer is one of the most common malignant tumors among women worldwide and has a high morbidity and mortality. This research aimed to identify hub genes and small molecule drugs for breast cancer by integrated bioinformatics analysis. After downloading multiple gene expression datasets from The Cancer Genome Atlas (TCGA) and Gene Expression Omnibus (GEO) database, 283 overlapping differentially expressed genes (DEGs) significantly enriched in different cancer-related functions and pathways were obtained using LIMMA, VennDiagram and ClusterProfiler packages of R. We then analyzed the topology of protein-protein interaction (PPI) network with overlapping DEGs and further obtained 6 hub genes (RRM2, CDC20, CCNB2, BUB1B, CDK1, and CCNA2) from the network via STRING and Cytoscape. Subsequently, we conducted genes expression verification, genetic alterations evaluation, immune infiltration prediction, clinicopathological parameters analysis, identification of transcriptional and post-transcriptional regulatory molecules, and survival analysis for these hub genes. Meanwhile, 29 possible drug candidates (e.g., Cladribine, Gallium nitrate, Alvocidib, 1ß-hydroxyalantolactone, Berberine hydrochloride, Nitidine chloride) were identified from DGIdb database and GSE85871 dataset. In addition, some transcription factors and miRNAs (e.g., E2F1, PTTG1, TP53, ZBTB16, hsa-miR-130a-3p, hsa-miR-204-5p) targeting hub genes were identified as key regulators in the progression of breast cancer. In conclusion, our study identified 6 hub genes and 29 potential drug candidates for breast cancer. These findings may advance understanding regarding the diagnosis, prognosis and treatment of breast cancer. 
1 Identification of hub genes and small molecule therapeutic

2 drugs related to breast cancer with comprehensive

3 bioinformatics analysis

4 Mingqian Hao, Wencong Liu, Chuanbo Ding, Xiaojuan Peng, Yue Zhang, Huiying Chen, Ling Dong,

5 Xinglong Liu, Yingchun Zhao, Xueyan Chen, Sadia Khatoon, Yinan Zheng

6 School of Chinese Medicinal Materials, Jilin Agricultural University, Changchun, Jilin, China

7

8 Corresponding Author:

9 Wencong Liu

10 No. 2888 Xincheng avenue, Changchun, Jilin, 130118, China

11 Email address: xiaodiliqian@163.com 


\section{Identification of hub genes and small molecule \\ 14 \\ 15 \\ therapeutic drugs related to breast cancer with comprehensive bioinformatics analysis}

16

17

18

19

20

21

22

23

24

25

26

27

28

29

30

31

32

33

34

35

36

37

38

39

40

41

42

43

44

45

46

47

Mingqian Hao, Wencong Liu, Chuanbo Ding, Xiaojuan Peng, Yue Zhang, Huiying Chen, Ling Dong, Xinglong Liu, Yingchun Zhao, Xueyan Chen, Sadia Khatoon, Yinan Zheng

School of Chinese Medicinal Materials, Jilin Agricultural University, Changchun, Jilin, China

Corresponding Author:

Wencong Liu

No. 2888 Xincheng avenue, Changchun, Jilin, 130118, China

Email address: xiaodiliqian@163.com

\section{Abstract}

Breast cancer is one of the most common malignant tumors among women worldwide and has a high morbidity and mortality. This research aimed to identify hub genes and small molecule drugs for breast cancer by integrated bioinformatics analysis. After downloading multiple gene expression datasets from The Cancer Genome Atlas (TCGA) and Gene Expression Omnibus (GEO) database, 283 overlapping differentially expressed genes (DEGs) significantly enriched in different cancer-related functions and pathways were obtained using LIMMA, VennDiagram and ClusterProfiler packages of $\mathrm{R}$. We then analyzed the topology of protein-protein interaction (PPI) network with overlapping DEGs and further obtained 6 hub genes (RRM2, CDC20, CCNB2, BUB1B, CDK1, and CCNA2) from the network via STRING and Cytoscape. Subsequently, we conducted genes expression verification, genetic alterations evaluation, immune infiltration prediction, clinicopathological parameters analysis, identification of transcriptional and post-transcriptional regulatory molecules, and survival analysis for these hub genes. Meanwhile, 29 possible drug candidates (e.g., Cladribine, Gallium nitrate, Alvocidib, 1及hydroxyalantolactone, Berberine hydrochloride, Nitidine chloride) were identified from DGIdb database and GSE85871 dataset. In addition, some transcription factors and miRNAs (e.g., E2F1, PTTG1, TP53, ZBTB16, hsa-miR-130a-3p, hsa-miR-204-5p) targeting hub genes were identified as key regulators in the progression of breast cancer. In conclusion, our study identified 6 hub genes and 29 potential drug candidates for breast cancer. These findings may advance understanding regarding the diagnosis, prognosis and treatment of breast cancer.

\section{Introduction}

Among cancers affecting females, breast cancer has a particularly high incidence, recurrence and mortality rate. Although encouraging progress has been made in the early diagnosis and systemic treatment over several decades, the overall 5 -year survival rate for patients with breast cancer is still low, and the incidence rate continues to increase annually (Bray et al. 2018; Waks \& Winer

Peer) reviewing PDF | (2020:06:49813:1:0:NEW 13 Aug 2020) 
48

49

50

51

52

53

54

55

56

57

58

59

60

61

62

63

64

65

66

67

68

69

70

71

72

73

74

75

76

77

78

79

80

81

82

83

84

85

86

2019). Breast cancer has become a serious public health problem all over the world, which brings great economic burdens to individuals and families. Thus, more efforts are required to find effective biomarkers for the early diagnosis, accurate prognosis, and targeted therapy of breast cancer.

High-throughput sequencing technology and bioinformatics analysis methods can reveal changes in the expression of a vast amount of genes and are also effective tools to identify candidate biomarkers for breast cancer research (Kulasingam \& Diamandis 2008). Liu et al. explored the expression and prognostic value of TDO2 in breast cancer using transcriptome data, and analyzed the correlation between TDO2 gene and tumor immune invasion, suggesting that TDO2 was a promising new immunotherapy target for breast cancer (Liu et al. 2020). Others, Li et al. studied the biological mechanism of BRCA1/2 mutant breast cancer and evaluated the diagnosis and prognosis values of key genes using bioinformatics methods ( $\mathrm{Li}$ et al. 2020b). In addition, to clarify the role of low-frequency mutated genes in breast cancer, Lusito and his colleagues used functional network construction, gene mutation analysis, hierarchical clustering and cancer module recognition to analyze the gene expression and mutation datasets of breast cancer (Lusito et al. 2019). Similarly, Wang et al. used research samples from The Cancer Genome Atlas (TCGA, https://www.cancer.gov/about-nci/organization/ccg/research/structural-genomics/tcga) and Gene Expression Omnibus (GEO, https://www.ncbi.nlm.nih.gov/geo/) database to analyze the pathogenesis and potential prognostic marker genes of breast cancer (Wang et al. 2019). In general, the majority of bioinformatics studies focused on how key genes affect the tumorigenesis and prognosis of breast cancer, with limited research on the systemic analysis of multi-gene and multi-pathway as well as potential drugs.

In the present study, we applied comprehensive bioinformatics methods, such as differentially expressed genes (DEGs) identification, protein-protein interaction (PPI) network integration, hub genes identification, correlation prediction between hub genes, determination of genetic alterations, Gene Ontology (GO) and Kyoto Encyclopedia of Genes and Genomes (KEGG) pathway enrichment, immune cell infiltrates evaluation, clinicopathological features analysis and survival analysis to process large-scale DNA microarrays and RNA-seq data from GEO, TCGA and other public databases so as to explore potential hub genes and biological pathways related to the occurrence, development and prognosis of breast cancer. We also explored the transcription factors (TFs) and miRNAs that regulate the transcriptional and post-transcriptional processes of hub genes, respectively. In addition, several small molecule drugs for breast cancer were obtained from the DGIdb drug relocation database (http://dgidb.org/) and GSE85871 dataset. Compared with similar publications, this study involved over 10,000 samples and more comprehensive analysis methods. Therefore, we may get more reliable and accurate information about breast cancer through this exploration. The workflow was explained in Fig. 1, and the details were provided in the materials and methods section.

\section{Materials \& Methods}

\section{Download of datasets and identification of DEGs}


875 independent gene expression profiles (GSE3744, GSE21422, GSE42568, GSE61304, and

88 GSE65194) based on GPL570 Platform (Affymetrix Human Genome U133 Plus 2.0 Array) were

89 downloaded from GEO database to identify DEGs. After normalization between arrays, we

90 investigated DEGs among each dataset with the threshold of $\mid \log 2$ FoldChange $(\log 2 \mathrm{FC}) \mid>1$

91 and adj.P.Val $<0.05$ using LIMMA package of R (Ritchie et al. 2015).

92 For validation, GEPIA2 online tool (http://gepia2.cancer-pku.cn/\#index) was used to analyze the

93 differential expression of TCGA Breast invasive carcinoma (BRCA) RNA-seq dataset composed

94 of 1,085 tumor samples and 112 normal samples according to the cut-off standard $(|\log 2 \mathrm{FC}|>1$

95 and $q$-value $<0.05)$. Lastly, the common results of TCGA BRCA dataset and GEO datasets were

96 selected as the overlapping DEGs of breast cancer, which could reduce the influences resulted

97 from the heterogeneity of the different datasets. Venn diagram and volcano plot were drawn by

98 VennDiagram and other packages of R. Table 1 listed the details of datasets.

99 Protein-protein interaction (PPI) network integration and hub genes screening

100 STRING (version 11.0; https://string-db.org/) is a biological database designed to analyze

101 functional interactions between proteins (Szklarczyk et al. 2019). In this study, we used STRING

102 to construct a PPI network with overlapping DEGs under the premise of an Interaction score of

103 0.7. Then, we utilized the cytoHubba plug-in of Cytoscape (version 3.7.2) which provided the

104 calculated results by maximal clique centrality (MCC), maximum neighborhood component

105 (MNC) and Degree methods to identify hub genes from the PPI network (Chin et al. 2014).

106 Verification of hub genes

107 The Oncomine database (https://www.oncomine.org) was used to verify the mRNA expression

108 of hub genes with the threshold of $P<0.05$ and fold change $>2$. Next, the Human Protein Atlas

109 database (HPA, https://www.proteinatlas.org) was used to validate the protein expression of

110 genes by immunohistochemistry data.

111 BC-GenExMiner (http://bcgenex.centregauducheau.fr/BC-GEM/GEM-Accueil.php) is a

112 statistical mining tool that contains published breast cancer transcription data (10,716 DNA

113 microarray samples and 4,712 RNA-seq samples) (Jézéquel et al. 2012). We performed

114 correlation analysis between hub genes in breast cancer using BC-GenExMiner and GEPIA2

115 online tool.

116 GO functional and KEGG pathway enrichment analysis

117 ClusterProfiler package of R can automatically classify biological terms and gene clusters (Yu et

118 al. 2012). To elucidate the biological characteristics of breast cancer-related genes, we performed

119 GO functional and KEGG pathway enrichment analysis by ClusterProfiler with $p$-value $<0.05$

120 and $q$-value $<0.05$.

121 Analysis of genetic alterations of hub genes

122 The cBioPortal for Cancer Genomics (cBioPortal; http://cbioportal.org) provides online

123 resources for the exploration, visualization and analysis of multidimensional cancer genomics

124 data (Gao et al. 2013). In this study, 6618 breast cancer samples from 13 related reports in

125 cBioPortal were used as research materials to explore genetic alterations connected with the

126 selected hub genes. Afterward, we utilized COSMIC (https://cancer.sanger.ac.uk/cosmic), the

Peer] reviewing PDF | (2020:06:49813:1:0:NEW 13 Aug 2020) 
127 most comprehensive resource for studying somatic mutation information in human cancer, to

128

129

130

131

132

133

134

135

136

137

138

139

140

141

142

143

144

145

146

147

148

149

150

151

152

153

154

155

156

157

158

159

160

161

162

163

164

165

analyze hub genes alterations in breast cancer (Forbes et al. 2010).

\section{Evaluation of clinicopathological characteristics and immune infiltration}

We used BC-GenExMiner to analyze the correlations between hub genes expression and clinicopathological variables such as Oestrogen receptor status (ER), Progesterone receptor status (PR), HER2 receptor status (HER2), Nodal status (N), Scarff Bloom \& Richardson grade status (SBR), Nottingham Prognostic Index status (NPI), Age status, P53 status, Basal-like and Triple negative breast cancer (TNBC) subtypes. $P<0.05$ was considered to be statistically significant.

TIMER (https://cistrome.shinyapps.io/timer/) is a comprehensive resource for systematic analysis of tumor-infiltrating immune cells across 32 different cancers from TCGA database (Li et al. 2017). In this experiment, we estimated the associations between hub genes expression and immune cell populations (B Cell, CD8+ T Cell, CD4+ T Cell, Macrophage, Neutrophil, and Dendritic Cell) in breast cancer using TIMER.

\section{Prediction of TFs-hub genes and miRNAs-hub genes interaction}

TRRUST (version 2, https://www.grnpedia.org/trrust/), a manually curated database of human and mouse transcriptional regulatory networks, was used to explore the TFs of hub genes (Han et al. 2018). Meanwhile, we used Encyclopedia of RNA Interactomes Platform (ENCORI, http://starbase.sysu.edu.cn/) to unearth the miRNAs targeting hub genes (Li et al. 2014). In addition, we utilized GEO2R online tool (https://www.ncbi.nlm.nih.gov/geo/geo2r/?acc=GSE97811) to analyze breast cancer differentially expressed miRNAs (DEmiRNAs) in GSE97811 dataset including 45 tumor tissues and 16 normal tissues. Then, we got miRNAs-hub genes interaction combined with miRNAstarget genes analysis and DEmiRNAs data. Finally, Cytoscape was used to visualize transcriptional and post-transcriptional regulatory networks.

\section{Survival analysis}

In this section, we applied the Kaplan-Meier Plotter (http://kmplot.com/analysis/) to evaluate prognostic information of previously identified hub genes and important reporter regulatory molecules (Nagy et al. 2018). The expression values of these genes were split into either high (expression value $\geq$ median) or low (expression value $<$ median). Hazard ratio (HR) was calculated to evaluate the association between genes expression and survival, and $p<0.05$ was considered statistically significant.

\section{Small molecule drugs analysis}

The DGIdb online tool (http://www.dgidb.org/) -an available resource containing drug-gene interaction information from more than 30 databases -was used to screen antineoplastic drugs targeting hub genes (Cotto et al. 2018). We also downloaded GSE85871 dataset, which is a gene expression data of MCF7 cells treated with 102 traditional Chinese medicine (TCM) ingredients recorded in the GEO database. LIMMA package of $\mathrm{R}$ was used to analyze the differential expression genes in each TCM ingredient treatment group compared with the untreated group 
166 (adj.p.val <0.05). Then, we evaluated the reversal effects of each TCM ingredient on

167 overlapping DEGs induced by breast cancer.

168

169

170

171

172

173

174

175

176

177

178

179

180

181

182

183

184

185

186

187

188

189

190

191

192

193

194

195

196

197

198

199

200

201

202

203

204

\section{Results}

\section{Dataset processing and DEGs acquisition}

After data normalization, the black lines of gene expression box plots of all samples in each single dataset were almost on the same level, which was an important marker to predict the reliability and accuracy of the experimental results (Fig. S1). Next, DEGs (1,738 in GSE3744, 2,430 in GSE21422, 3,116 in GSE42568, 990 in GSE61304 and 4,181 in GSE65194) were identified (Fig. 2A-2E; Table S1-S5), and 302 integrated DEGs (110 up- and 192 downregulated) from 5 GEO datasets were found (Fig. 2F and 2G). Similarly, we identified 3,559 DEGs from TCGA BRCA dataset with the cut-off criteria of $|\log 2 \mathrm{FC}|>1$ and $q$-value $<$ 0.05 (Table S6). Then, 283 overlapping DEGs which might play promoting or inhibitory roles in breast cancer progression were confirmed from the analysis results of GEO datasets and TCGA BRCA dataset (Fig. 2H and 2I).

\section{PPI network construction and hub genes filtering}

The PPI network around proteins encoded by 283 overlapping DEGs was constructed using STRING (Fig. S2A, Table S7). We found that 165 of the 283 overlapping DEGs were related to each other and were visualized using Cytoscape -165 nodes and 1,861 edges were included in PPI network and 6 hub genes (RRM2, CDC20, CCNB2, BUB1B, CDK1, and CCNA2) based on MCC, MNC and Degree methods were identified (Fig. S2B). Notably, these hub genes were all up-regulated in overlapping DEGs (Table S1-S6) and might play important roles in the pathogenesis of breast cancer.

\section{Verification of hub genes}

Based on the large-scale breast cancer-related data in the Oncomine database, we confirmed that hub genes were significantly up-regulated in multiple cancer types, including Breast Cancer, Brain and CNS Cancer, Lymphoma, Lung Cancer, and so on (Fig. 3A). Also, immunohistochemistry staining data obtained from the HPA database demonstrated the upregulated expression of proteins encoded by RRM2, CDC20, CCNB2, CDK1 and CCNA2 (Fig. 3B-3K). However, we did not find the association between BUB1B and breast cancer in HPA database. According to the current analysis, we predicted that BUB1B might also be associated with breast cancer, but experimental data were needed to confirm this specific connection. Meanwhile, CDK1, CCNA2, CCNB2, BUB1B and CDC20 were classified as cancer-related genes, and RRM2 was an FDA approved drug target. The data of BC-GenExMiner and GEPIA2 both confirmed a powerful correlation among hub genes (Fig. S3), suggesting that these genes might be the functional partners in breast cancer.

\section{GO function enrichment and KEGG pathway analysis}

The GO function annotations of overlapping DEGs were mainly classified into biological processes (BP), cell component (CC) and molecular function (MF). As for BP, up-regulated overlapping DEGs were significantly related to mitotic nuclear division, organelle fission, and 
205 regulation of chromosome segregation, which was consistent with the biological characteristics

206 of the abnormally rapid proliferation of breast cancer cells. And the down-regulated genes were 207 closely related to regulation of cellular response to growth factor stimulus, temperature

208 homeostasis, retinoid metabolic process and regulation of vasculature development. Within CC, 209 the up-regulated genes were remarkably correlated to spindle, chromosome, centromeric region, 210 condensed chromosome kinetochore, and midbody, whereas the down-regulated genes were 211 related to collagen-containing extracellular matrix and sarcolemma. MF analysis displayed the

212 up-regulated genes were involved in microtubule binding, microtubule motor activity, and

213 cyclin-dependent protein serine/threonine kinase regulator activity, whereas the down-regulated

214 genes were mainly enriched in glycosaminoglycan binding, heparin binding, extracellular matrix 215 structural constituent and growth factor binding (Fig. 4A and 4B; Table S8 and S9).

216 KEGG pathway analysis showed the up- and down-regulated overlapping DEGs were all 217 significantly attached to Cell cycle, Oocyte meiosis, Tyrosine metabolism, ECM-receptor 218 interaction, Progesterone-mediated oocyte maturation, p53 signaling pathway, PPAR signaling 219 pathway and Phenylalanine metabolism (Fig. 4C). Furthermore, the related pathways of hub 220 genes included Cell cycle, Oocyte meiosis, Progesterone-mediated oocyte maturation, and p53 221 signaling pathway (Fig. 4C). Table S10 presented the detailed results of KEGG enrichment 222 analysis.

\section{Genetic alterations of hub genes}

224 As a result, there were nearly 2.1\% (CDC20, CDK1), 1.2\% (RRM2), 0.9\% (BUB1B), $0.8 \%$

225 (CCNA2), 0.7\% (CCNB2) of breast cancer samples included in cBioPortal had genetic changes.

226 Amplification was the most common genetic alterations among 6 hub genes. And deep deletion

227 was another major genetic change among five hub genes (RRM2, CCNB2, BUB1B, CDK1, 228 CCNA2). While genetic alterations in RRM2, CDC20, BUB1B, CDK1 and CCNA2 were related 229 to missense mutation (Fig. S4A). In the query results of COSMIC database, we found that all 230 hub genes had missense mutations. RRM2, CCNB2, CDK1, BUB1B and CDC20 experienced 231 synonymous substitutions. CCNA2 and BUB1B also had frameshift deletions. Specifically, these 232 hub genes had mutations such as $\mathrm{A}>\mathrm{G}, \mathrm{A}>\mathrm{T}, \mathrm{C}>\mathrm{T}, \mathrm{C}>\mathrm{G}, \mathrm{G}>\mathrm{A}, \mathrm{G}>\mathrm{T}$ at nucleotide level 233 (Fig. S4B).

\section{Clinicopathological characteristics and immune infiltration}

235 We investigated the relevance of 6 hub genes and clinicopathological features using BC236 GenExMiner (Fig. S5-S7). Data analysis showed that higher expression of hub genes was found 237 in higher NPI and SBR grade $(p<0.001)$. And the expression of these hub genes was 238 significantly higher in ER-, PR-, HER2+, Nodal+, P53-mutated, Basal-like and TNBC clinical 239 subtypes of breast cancer. Surprisingly, significantly increased expression of hub genes was 240 found in patients not more than 51 years old $(p<0.001)$. Moreover, our current results 241 demonstrated that hub genes were correlated with 6 types of immune cell infiltrates (B Cell, 242 CD8+ T Cell, CD4+ T Cell, Macrophage, Neutrophil and Dendritic Cell) with various degrees 243 based on the TIMER database (Fig. S8).

244 Prediction of TF-hub genes and miRNAs-hub genes interaction 
245 We screened the potential regulatory relationships between TFs and hub genes via TRRUST 246 database to further study the functional roles of hub genes. A total of 19 associations between 17

247 TFs and 6 hub genes were shown (Fig. 5A). E2F1 could activate the transcriptional process of

248 RRM2 and CDK1. In contrast, TP53 inhibited the expression of CDK1 and CCNB2. Noticeably, 249 PTTG1 gene, the transcriptional promoter of CDK1, was up-regulated in breast cancer; ZBTB16

250

251

252

253

254

255

256

257

258

259

260

261

262

263

264

265

266

267

268

269

270

271

272

273

274

275

276

277

278

279

280

281

282

283

284

gene was the transcriptional suppressor of CCNA2, and its expression was down-regulated in overlapping DEGs. Additionally, we obtained 273 targeted miRNAs with regulatory effects on hub genes using ENCORI online tool, and identified 103 DEmiRNAs (58 up- and 45 downregulated; Table S11) from GSE97811 dataset with $|\log 2 \mathrm{FC}|>1$ and adj.P.Val $<0.05$. As a result, 41 associations between 30 miRNAs and 4 hub genes (RRM2, CDK1, CCNA2, and BUB1B) were found from targeted miRNAs analysis and DEmiRNAs data (Fig. 5B). The top 2 hub genes with the most miRNAs targets were RRM2 and CCNA2. In addition, hsa-miR-340-5p, hsa-miR-130a-3p, hsa-miR-200b-3p, hsa-miR-200c-3p, hsa-miR-204-5p, hsa-miR-219a-5p, hsamiR-27a-3p, hsa-miR-27b-3p, hsa-miR-301a-3p and hsa-miR-429 were the top 10 miRNAs with the most target genes. Unfortunately, we only used TRRUST and ENCORI databases, as well as limited samples of GSE97811 dataset to analyze TFs and miRNAs targeting hub genes, which may potentially limit the completeness of this study.

\section{Survival analysis of hub genes}

We then analyzed the prognostic information of 6 hub genes using Kaplan-Meier Plotter. The result demonstrated that breast cancer patients with higher hub genes expression had worse OS, relapse-free survival (RFS) and distant metastasis-free survival (DMFS) (Fig. 6A-6C).

Furthermore, we performed prognostic analysis on the important reporter regulatory molecules and found that higher E2F1 and PTTG1 expression predicted worse OS. In contrast, higher expression of ZBTB16, hsa-miR-130a-3p and hsa-miR-204-5p was significantly associated with better OS in breast cancer (Fig. S9A). The remaining reporter regulatory molecules (e.g., TP53, hsa-miR-340-5p, hsa-miR-200b-3p, hsa-miR-200c-3p, hsa-miR-219a-5p, hsa-miR-27a-3p, hsamiR-27b-3p, hsa-miR-301a-3p, hsa-miR-429) had no statistically significant correlation with OS $(P>0.05$; Fig. S9B).

\section{Small molecule drugs analysis}

Regarding 6 hub genes as potential therapeutic targets for breast cancer, we identified several antineoplastic drugs based on the DGIdb database. At present, only RRM2, CDK1 and CCNA2 were identified as tumor therapeutic targets. Therefore, we speculated the other three genes (CCNB2, BUB1B, CDC20) might be the novel targets in the future. Statistical analysis revealed that 21 candidate drugs such as Cladribine, Gallium nitrate, Dinaciclib, Alvocidib and Suramin targeted RRM2, CDK1 and CCNA2 (Table 2). More experimental data are needed to further confirm the potential of these drug candidates in the treatment of breast cancer.

In addition, we identified 8 TCM ingredients (1 $\beta$-hydroxyalantolactone, Andrographolide, Berberine hydrochloride, Britanin, Hyodeoxycholic acid, Japonicone A, Nitidine chloride and Tanshinone IIA) that reversed breast cancer-induced gene expression from GSE85871 dataset. Japonicone A reversed the expression of 87 overlapping DEGs, including 6 hub genes, and its

Peer) reviewing PDF | (2020:06:49813:1:0:NEW 13 Aug 2020) 
285 potential therapeutic effects on breast cancer were related to Cell cycle, Oocyte meiosis, p53 286 signaling pathway, Progesterone-mediated oocyte maturation, Viral carcinogenesis, HTLV-I 287 infection, Pathways in cancer and TGF-beta signaling pathway (Fig. 7A). Also, Nitidine 288 chloride, Berberine hydrochloride, 1 $\beta$-hydroxyalantolactone, Britanin and Tanshinone IIA 289 reversed the expression of 37, 35, 31, 30 and 19 overlapping DEGs, respectively, and the 290 potential therapeutic effects of these ingredients on breast cancer were related to biological 291 pathways such as Cell cycle, Oocyte meiosis, p53 signaling pathway and Progesterone-mediated 292 oocyte maturation (Fig. 7B-7F). Details of TCM ingredients were shown in Table S12.

293 Discussion

294

295

296

297

298

299

300

301

302

303

304

305

306

307

308

309

310

311

312

313

314

315

316

317

318

319

320

321

322

323

324

Although the detection and treatment of breast cancer have improved, it is still one of the most prevalent malignant tumors with the highest increase in prevalence among women (Ghoncheh et al. 2016). The diagnosis, treatment and prognosis of breast cancer have always been concerned with the world. Gene expression profiles are widely used to explore the molecular mechanisms related to tumorigenesis, which have provided valuable reference and information for clinical applications (Mohr et al. 2002).

In this study, we identified 283 overlapping DEGs (105 up- and 178 down-regulated) and 6 hub genes (RRM2, CDC20, CCNB2, BUB1B, CDK1, CCNA2) associated with breast cancer tumorigenesis and progression based on multiple datasets. By integrating the Oncomine, HPA, GEPIA2 and BC-GenExMiner databases, we confirmed that hub genes were over-expressed at mRNA and protein levels in breast cancer tissues compared with normal and non-cancerous tissues, and there was a powerful correlation between these genes, suggesting that hub genes were potential functional partners closely related to breast cancer. Furthermore, higher expression of hub genes was found in ER-, PR-, HER2+, Nodal+, Basal-like, P53-mutated and TNBC clinical subtypes of breast cancer, and there was a higher hub genes expression in patients not more than 51 years old. We also discovered that the over-expression of each hub gene was associated with poor OS, RFS and DMFS among patients with breast cancer, suggesting that these genes might be potential prognostic biomarkers and promote the progression of breast cancer. Further, we found the expression of hub genes was significantly correlated with immune cell infiltrates and purity, implicating that these hub genes played important roles in manipulating breast cancer immune microenvironment. KEGG enrichment analysis showed that overlapping DEGs including 6 hub genes were significantly associated with Cell cycle and Oocyte meiosis biological pathways, and overlapping DEGs were also involved in Tyrosine metabolism, ECM-receptor interaction, Progesterone-mediated oocyte maturation and PPAR signaling pathways. To our knowledge, abnormal regulations of cell cycle and cell growth were the major causes of tumorigenesis (Zhuang et al. 2015). In the current study, we found that two important pathways related to cell growth and apoptosis -Cell cycle and Oocyte meiosis -were dysregulated in breast cancer. In addition, ECM-receptor interaction pathway played important roles in tumor shedding, adhesion, degradation, movement and hyperplasia. So far, some studies have shown that ECM receptor interaction pathway was closely related to breast cancer, colorectal cancer, head and neck cancer 
325

326

327

328

329

330

331

332

333

334

335

336

337

338

339

340

341

342

343

344

345

346

347

348

349

350

351

352

353

354

355

356

357

358

359

360

361

362

363

and other human tumors (Bao et al. 2019; Islam et al. 2018; Rahman et al. 2019). Gasco et al. concluded that molecular pathological analysis of specific components of p53 signaling pathway may be helpful for the diagnosis and prognosis of breast cancer (Gasco et al. 2002). In addition, it has been found that signal transduction pathways such as Tyrosine metabolism, Progesteronemediated oocyte maturation and PPAR signaling pathway may also be associated with the occurrence of human cancers (Chen et al. 2012; Liu \& Ye 2017; Pietras et al. 1995). These pathways provided insights into the molecular mechanisms of breast cancer initiation and development.

Accumulating studies have demonstrated that CDK1, CCNB2, CCNA2, CDC20 and BUB1B, as genes related to cell cycle, are involved in the occurrence and development of tumors. CDK1, also known as CDC2, plays an important role in the precise cell division (Kang et al. 2014). Inhibiting the expression of CDK1 can suppress tumor cells growth and induce apoptosis in TNBC clinical subtype of breast cancer (Liu et al. 2014). In addition, high expression of CDK1 led to worse 5-year RFS in breast cancer patients (Kim et al. 2008), similar to the experimental results (Fig. 6B). As an important component in cell cycle regulation, CCNB2 seems to functions as the oncogene and independent prognostic factor for survival in patients with breast cancer (Albulescu 2013). Tang et al. proved that there was a significant correlation between CCNB2 and molecular subtypes of breast cancer (Fig. S6A) (Tang et al. 2018). CCNA2, a key regulator of cell cycle, could promote the transformation and progression of cancer (He et al. 2017). Gao et al. found the over-expression of CCNA2 in breast cancer was related to the unfavorable prognosis (Gao et al. 2014), similar to our current findings (Fig. 6). We understand that there have been previous reports that have associated CDC20 over-expression with tumor progression and poor prognosis of breast cancer, indicating that CDC20 may be a useful marker for monitoring breast cancer progression (Sewart \& Hauf 2017). Similarly, BUB1B played a pivotal role in the proliferation and progression of many tumors (Takagi et al. 2013). RRM2, a breast cancer hub gene participated in phenylalanine metabolic pathway in our study, was closely linked to tumor growth, invasion, angiogenesis, tumor metastasis and other cellular functions, as well as the prognosis of breast cancer patients (Bell et al. 2017; Chen et al. 2019). It has been previously reported that RRM2 expression was associated with the resistance of tumorigenic breast cancer cells to chemotherapy (Shah et al. 2015). Taken together, our findings were consistent with other previous studies that 6 hub genes may serve as predictive biomarkers for diagnosis and prognosis of patients with breast cancer.

We also found that several reporter regulatory molecules (e.g., E2F1, TP53, PTTG1, ZBTB16, hsa-miR-340-5p, hsa-miR-130a-3p, hsa-miR-200b-3p, hsa-miR-204-5p) regulated the transcription or post-transcription of hub genes associated with major biological processes and pathways in breast cancer, and they were also related to the OS of breast cancer patients. E2F1 and ZBTB16 have been proved to belong to tumor-suppressive genes (Wasim et al. 2010; Worku et al. 2008). In contrast, PTTG1 and mutated TP53 promoted the proliferation of tumor cells (Fu et al. 2018; Gasco et al. 2002). 
364 Breast cancer also shows changes in the expressions of noncoding RNAs (e.g., miRNAs,

365

366

367

368

369

370

371

372

373

374

375

376

377

378

379

380

381

382

383

384

385

386

387

388

389

390

391

392

393

394

395

396

397

398

399

400

401 additional elements). Several studies have previously proven that miRNAs played vital roles in many biological processes, including cell growth, differentiation, metabolism, apoptosis and signal transduction. For instance, Ma et al. demonstrated that miR-219-5p inhibited the cell proliferation and cell cycle distribution of ESCC cells by inhibiting the expression of CCNA2, highlighting the role of miR-219-5p and CCNA2 in cell cycle and tumor growth (Ma 2019). Liang et al. confirmed the over-expression of miR-204-5p not only inhibited the high expression of RRM2 in breast cancer cells but also inhibited cell migration and invasion of breast cancer (Liang et al. 2019). The above conclusions further demonstrated the miRNAs identified in this study were promising biomarkers for breast cancer. Recent publications described that additional elements including REP522, D20S16, HERVKC4-INT and HERV1_LTRc were also abnormally expressed in ER+/HER2- breast cancer, showing that it is necessary to further strengthen the study of these additional elements related to cancer (Karakülah et al. 2019; Yandım \& Karakülah 2019).

Next, we further identified 21 anti-tumor drugs (e.g., Cladribine, Gallium nitrate, Dinaciclib, Alvocidib, Suramin) targeting RRM2, CDK1 and CCNA2 (Table 2). Nevertheless, whether these drugs could exert therapeutic effects on breast cancer by inhibiting the over-expression of RRM2, CDK1 and CCNA2, or whether CCNB2, BUB1B and CDC20 are promising therapeutic targets still need to be supported by further research. In addition, we identified $8 \mathrm{TCM}$ ingredients (1 $\beta$-hydroxyalantolactone, Andrographolide, Berberine hydrochloride, Britanin, Hyodeoxycholic acid, Japonicone A, Nitidine chloride and Tanshinone IIA) that reversed breast cancer-induced overlapping DEGs expression. Previous limited studies have reported that some of TCM ingredients mentioned above affected the cell cycle of tumor cells (Du et al. 2015; Li et al. 2020a; Lu 2009; Pan et al. 2011; Wang et al. 2016; Zou et al. 2017), similar to our current study that these ingredients inhibited proliferation as well as promoted apoptosis of breast cancer cells through more than one biological pathway (Fig. 7).

\section{Conclusions}

In the current study, we obtained 283 overlapping DEGs and 6 hub genes (RRM2, CDC20, CCNB2, BUB1B, CDK1, and CCNA2) related to the occurrence and development of breast cancer via comprehensive bioinformatics analysis. Furthermore, we considered the associations between hub genes expression and clinicopathological factors (e.g., age, subtypes) of patients with breast cancer. The study also established TFs-hub genes and miRNAs-hub genes networks, and found several reporter regulatory molecules (e.g., E2F1, PTTG1, TP53, ZBTB16, hsa-miR130a-3p, hsa-miR-204-5p) significantly related to the progression and prognosis of breast cancer. Meanwhile, 29 small molecule drugs with potential therapeutic effects for breast cancer were identified from the DGIdb database and GSE85871 dataset. In summary, our research provided further clues for breast cancer therapeutic drugs and biomarkers.

\section{Abbreviations}




\begin{tabular}{|c|c|}
\hline DEGs & differentially expressed genes \\
\hline GEO & Gene Expression Omnibus \\
\hline TCGA & The Cancer Genome Atlas \\
\hline PPI & protein-protein interaction \\
\hline $\mathrm{GO}$ & Gene Ontology \\
\hline KEGG & Kyoto Encyclopedia of Genes and Genomes \\
\hline TFs & transcription factors \\
\hline TCM & traditional Chinese medicine \\
\hline $\log 2 \mathrm{FC}$ & log2FoldChange \\
\hline $\mathrm{BRCA}$ & Breast invasive carcinoma \\
\hline $\mathrm{MCC}$ & maximal clique centrality \\
\hline $\mathrm{MNC}$ & maximum neighbourhood component \\
\hline HPA & Human Protein Atlas database \\
\hline ER & Oestrogen receptor status \\
\hline PR & Progesterone receptor status \\
\hline HER2 & HER2 receptor status \\
\hline SBR & Scarff Bloom \& Richardson grade status \\
\hline NPI & Nottingham Prognostic Index status \\
\hline TNBC & Triple negative breast cancer \\
\hline ENCORI & Encyclopedia of RNA Interactomes platform \\
\hline DEmiRNAs & differentially expressed miRNAs \\
\hline HR & hazard ratio \\
\hline OS & overall survival \\
\hline RFS & relapse-free survival \\
\hline DMFS & distant metastasis-free survival \\
\hline
\end{tabular}

402 References 
403

404

405

406

407

408

409

410

411

412

413

414

415

416

417

418

419

420

421

422

423

424

425

426

427

428

429

430

431

432

433

434

435

436

437

438

439

440

441

442

443

444

445

446

447

448

449

450

451

452

453

454

455

456

Albulescu R. 2013. Elevated cyclin B2 expression in invasive breast carcinoma is associated with unfavorable clinical outcome. Biomark Med 7:203.

Bao Y, Wang L, Shi L, Yun F, Liu X, Chen Y, Chen C, Ren Y, and Jia Y. 2019. Transcriptome profiling revealed multiple genes and ECM-receptor interaction pathways that may be associated with breast cancer. Cell Mol Biol Lett 24:38. 10.1186/s11658-019-0162-0

Bell R, Barraclough R, and Vasieva O. 2017. Gene Expression Meta-Analysis of Potential Metastatic Breast Cancer Markers. Current Molecular Medicine 17. 10.2174/1566524017666170807144946

Bray F, Ferlay J, Soerjomataram I, Siegel RL, Torre LA, and Jemal A. 2018. Global cancer statistics 2018: GLOBOCAN estimates of incidence and mortality worldwide for 36 cancers in 185 countries. CA Cancer J Clin 68:394-424. 10.3322/caac.21492

Chen W-x, Yang L-g, Xu L-y, Cheng L, Qian Q, Sun L, and Zhu Y-I. 2019. Bioinformatics analysis revealing prognostic significance of RRM2 gene in breast cancer. Bioscience reports 39.

Chen YZ, Xue JY, Chen CM, Yang BL, Xu QH, Wu F, Liu F, Ye X, Meng X, Liu GY, Shen ZZ, Shao ZM, and Wu J. 2012. PPAR signaling pathway may be an important predictor of breast cancer response to neoadjuvant chemotherapy. Cancer Chemother Pharmacol 70:637-644. 10.1007/s00280-012-1949-0

Chin C-H, Chen S-H, Wu H-H, Ho C-W, Ko M-T, and Lin C-Y. 2014. cytoHubba: identifying hub objects and sub-networks from complex interactome. BMC systems biology 8:S11.

Cotto KC, Wagner AH, Feng Y-Y, Kiwala S, Coffman AC, Spies G, Wollam A, Spies NC, Griffith OL, and Griffith M. 2018. DGIdb 3.0: a redesign and expansion of the drug-gene interaction database. Nucleic acids research 46:D1068-D1073.

Du Y, Gong J, Tian X, Yan X, Guo T, Huang M, Zhang B, Hu X, Liu H, and Wang Y. 2015. Japonicone A inhibits the growth of non-small cell lung cancer cells via mitochondria-mediated pathways. Tumor Biology 36:7473-7482.

Forbes SA, Bindal N, Bamford S, Cole C, Kok CY, Beare D, Jia M, Shepherd R, Leung K, and Menzies A. 2010. COSMIC: mining complete cancer genomes in the Catalogue of Somatic Mutations in Cancer. Nucleic acids research 39:D945-D950.

Fu D, Zhang Y, and Cui H. 2018. Long noncoding RNA CCAT2 is activated by E2F1 and exerts oncogenic properties by interacting with PTTG1 in pituitary adenomas. Am J Cancer Res 8:245255.

Gao J, Aksoy BA, Dogrusoz U, Dresdner G, Gross B, Sumer SO, Sun Y, Jacobsen A, Sinha R, and Larsson E. 2013. Integrative analysis of complex cancer genomics and clinical profiles using the cBioPortal. Sci Signal 6:pl1-pl1.

Gao T, Han Y, Yu L, Ao S, Li Z, and Ji J. 2014. CCNA2 is a prognostic biomarker for ER+ breast cancer and tamoxifen resistance. PLOS ONE 9:e91771. 10.1371/journal.pone.0091771

Gasco M, Shami S, and Crook T. 2002. The p53 pathway in breast cancer. Breast cancer research 4:70.

Ghoncheh M, Pournamdar Z, and Salehiniya H. 2016. Incidence and Mortality and Epidemiology of Breast Cancer in the World. Asian Pacific Journal of Cancer Prevention 17:43-46. 10.7314/apjcp.2016.17.S3.43

Han H, Cho JW, Lee S, Yun A, Kim H, Bae D, Yang S, Kim CY, Lee M, Kim E, Lee S, Kang B, Jeong D, Kim Y, Jeon HN, Jung H, Nam S, Chung M, Kim JH, and Lee I. 2018. TRRUST v2: an expanded reference database of human and mouse transcriptional regulatory interactions. Nucleic Acids Res 46:D380-d386. 10.1093/nar/gkx1013

He Y, Liu J, Zhao Z, and Zhao H. 2017. Bioinformatics analysis of gene expression profiles of esophageal squamous cell carcinoma. Diseases of the Esophagus 30.

Islam T, Rahman R, Gov E, Turanli B, Gulfidan G, Haque A, Arga KY, and Haque Mollah N. 2018. Drug Targeting and Biomarkers in Head and Neck Cancers: Insights from Systems Biology Analyses. Omics 22:422-436. 10.1089/omi.2018.0048

Jézéquel P, Campone M, Gouraud W, Guérin-Charbonnel C, Leux C, Ricolleau G, and Campion L. 2012. bc-GenExMiner: an easy-to-use online platform for gene prognostic analyses in breast cancer. Breast Cancer Research and Treatment 131:765-775.

Kang J, Sergio CM, Sutherland RL, and Musgrove EA. 2014. Targeting cyclin-dependent kinase 1 (CDK1) but not CDK4/6 or CDK2 is selectively lethal to MYC-dependent human breast cancer cells. BMC Cancer 14:32.

Peer] reviewing PDF | (2020:06:49813:1:0:NEW 13 Aug 2020) 
457

458

459

460

461

462

463

464

465

466

467

468

469

470

471

472

473

474

475

476

477

478

479

480

481

482

483

484

485

486

487

488

489

490

491

492

493

494

495

496

497

498

499

500

501

502

503

504

505

506

507

508

509

510

Karakülah G, Arslan N, Yandım C, and Suner A. 2019. TEffectR: an R package for studying the potential effects of transposable elements on gene expression with linear regression model. PeerJ 7:e8192. 10.7717/peerj.8192

Kim SJ, Nakayama S, Miyoshi Y, Taguchi T, Tamaki Y, Matsushima T, Torikoshi Y, Tanaka S, Yoshida T, Ishihara $\mathrm{H}$, and Noguchi S. 2008. Determination of the specific activity of CDK1 and CDK2 as a novel prognostic indicator for early breast cancer. Ann Oncol 19:68-72. 10.1093/annonc/mdm358

Kulasingam V, and Diamandis EP. 2008. Strategies for discovering novel cancer biomarkers through utilization of emerging technologies. Nature clinical practice Oncology 5:588-599.

Li J-H, Liu S, Zhou H, Qu L-H, and Yang J-H. 2014. starBase v2.0: decoding miRNA-ceRNA, miRNAncRNA and protein-RNA interaction networks from large-scale CLIP-Seq data. Nucleic acids research 42:D92-D97. 10.1093/nar/gkt1248

Li K, Zhou Y, Chen Y, Zhou L, and Liang J. 2020a. A novel natural product, britanin, inhibits tumor growth of pancreatic cancer by suppressing nuclear factor-kappaB activation. Cancer Chemother Pharmacol 85:699-709. 10.1007/s00280-020-04052-w

Li T, Fan J, Wang B, Traugh N, Chen Q, Liu JS, Li B, and Liu XS. 2017. TIMER: A Web Server for Comprehensive Analysis of Tumor-Infiltrating Immune Cells. Cancer research 77:e108-e110. 10.1158/0008-5472.Can-17-0307

Li Y, Zhou X, Liu J, Yin Y, Yuan X, Yang R, Wang Q, Ji J, and He Q. 2020b. Differentially expressed genes and key molecules of BRCA1/2-mutant breast cancer: evidence from bioinformatics analyses. PeerJ 8. 10.7717/peerj.8403

Liang WH, Li N, Yuan ZQ, Qian XL, and Wang ZH. 2019. DSCAM-AS1 promotes tumor growth of breast cancer by reducing miR-204-5p and up-regulating RRM2. Mol Carcinog 58:461-473. 10.1002/mc.22941

Liu $\mathrm{H}$, and Ye H. 2017. Screening of the prognostic targets for breast cancer based co-expression modules analysis. Mol Med Rep 16:4038-4044. 10.3892/mmr.2017.7063

Liu Q, Zhai J, Kong X, Wang X, Wang Z, Fang Y, and Wang J. 2020. Comprehensive Analysis of the Expression and Prognosis for TDO2 in Breast Cancer. Mol Ther Oncolytics 17:153-168. 10.1016/j.omto.2020.03.013

Liu Y, Zhu YH, Mao CQ, Dou S, Shen S, Tan ZB, and Wang J. 2014. Triple negative breast cancer therapy with CDK1 siRNA delivered by cationic lipid assisted PEG-PLA nanoparticles. $J$ Control Release 192:114-121. 10.1016/j.jconrel.2014.07.001

Lu. 2009. Experimental study of the anti-cancer mechanism of tanshinone IIA against human breast cancer. International Journal of Molecular Medicine 24. 10.3892/ijmm_00000291

Lusito E, Felice B, D'Ario G, Ogier A, Montani F, Di Fiore PP, and Bianchi F. 2019. Unraveling the role of low-frequency mutated genes in breast cancer. Bioinformatics 35:36-46.

Ma Q. 2019. MiR-219-5p suppresses cell proliferation and cell cycle progression in esophageal squamous cell carcinoma by targeting CCNA2. Cell Mol Biol Lett 24:4. 10.1186/s11658-0180129-6

Mohr S, Leikauf GD, Keith G, and Rinn BH. 2002. Microarrays as Cancer Keys: An Array of Possibilities. Journal of Clinical Oncology 20:3165-3175. 10.1200/jco.2002.12.073

Nagy Á, Lánczky A, Menyhárt O, and Győrffy B. 2018. Validation of miRNA prognostic power in hepatocellular carcinoma using expression data of independent datasets. Scientific Reports 8:9227. 10.1038/s41598-018-27521-y

Pan X, Han H, Wang L, Yang L, Li R, Li Z, Liu J, Zhao Q, Qian M, and Liu M. 2011. Nitidine chloride inhibits breast cancer cells migration and invasion by suppressing c-Src/FAK associated signaling pathway. Cancer letters 313:181-191.

Pietras RJ, Arboleda J, Reese DM, Wongvipat N, Pegram MD, Ramos L, Gorman CM, Parker MG, Sliwkowski MX, and Slamon DJ. 1995. HER-2 tyrosine kinase pathway targets estrogen receptor and promotes hormone-independent growth in human breast cancer cells. Oncogene 10:24352446.

Rahman MR, Islam T, Gov E, Turanli B, Gulfidan G, Shahjaman M, Banu NA, Mollah MNH, Arga KY, and Moni MA. 2019. Identification of Prognostic Biomarker Signatures and Candidate Drugs in Colorectal Cancer: Insights from Systems Biology Analysis. Medicina (Kaunas) 55. 10.3390/medicina55010020

Peer) reviewing PDF | (2020:06:49813:1:0:NEW 13 Aug 2020) 
511 Ritchie ME, Phipson B, Wu D, Hu Y, Law CW, Shi W, and Smyth GK. 2015. limma powers differential

512

513

514

515

516

517

518

519

520

521

522

523

524

525

526

527

528

529

530

531

532

533

534

535

536

537

538

539

540

541

542

543

544

545

546

547

548

549

550 expression analyses for RNA-sequencing and microarray studies. Nucleic acids research 43:e47e47.

Sewart K, and Hauf S. 2017. Different functionality of Cdc20 binding sites within the mitotic checkpoint complex. Current Biology 27:1213-1220.

Shah KN, Wilson EA, Malla R, Elford HL, and Faridi JS. 2015. Targeting ribonucleotide reductase M2 and NF-kB activation with Didox to circumvent tamoxifen resistance in breast cancer. Molecular Cancer Therapeutics 14:2411-2421.

Szklarczyk D, Gable AL, Lyon D, Junge A, Wyder S, Huerta-Cepas J, Simonovic M, Doncheva NT, Morris $\mathrm{JH}$, and Bork P. 2019. STRING v11: protein-protein association networks with increased coverage, supporting functional discovery in genome-wide experimental datasets. Nucleic acids research 47:D607-D613.

Takagi K, Miki Y, Shibahara Y, Nakamura Y, Ebata A, Watanabe M, Ishida T, Sasano H, and Suzuki T. 2013. BUB1 Immunolocalization in Breast Carcinoma: Its Nuclear Localization as a Potent Prognostic Factor of the Patients. Hormones and Cancer 4:92-102. 10.1007/s12672-012-0130-x

Tang J, Kong D, Cui Q, Wang K, Zhang D, Gong Y, and Wu G. 2018. Prognostic Genes of Breast Cancer Identified by Gene Co-expression Network Analysis. Front Oncol 8:374. 10.3389/fonc.2018.00374

Waks AG, and Winer EP. 2019. Breast cancer treatment: a review. Jama 321:288-300.

Wang CCN, Li CY, Cai JH, Sheu PC, Tsai JJP, Wu MY, Li CJ, and Hou MF. 2019. Identification of Prognostic Candidate Genes in Breast Cancer by Integrated Bioinformatic Analysis. J Clin Med 8. 10.3390/jcm8081160

Wang H-Y, Yu H-Z, Huang S-M, and Zheng Y-L. 2016. p53, Bcl-2 and cox-2 are involved in berberine hydrochloride-induced apoptosis of HeLa229 cells. Molecular Medicine Reports 14:3855-3861. 10.3892/mmr.2016.5696

Wasim M, Carlet M, Mansha M, Greil R, Ploner C, Trockenbacher A, Rainer J, and Kofler R. 2010. PLZF/ZBTB16, a glucocorticoid response gene in acute lymphoblastic leukemia, interferes with glucocorticoid-induced apoptosis. J Steroid Biochem Mol Biol 120:218-227. 10.1016/j.jsbmb.2010.04.019

Worku D, Jouhra F, Jiang GW, Patani N, Newbold RF, and Mokbel K. 2008. Evidence of a tumour suppressive function of E2F1 gene in human breast cancer. Anticancer Res 28:2135-2139.

Yandım C, and Karakülah G. 2019. Dysregulated expression of repetitive DNA in ER+/HER2- breast cancer. Cancer Genet 239:36-45. 10.1016/j.cancergen.2019.09.002

Yu G, Wang L-G, Han Y, and He Q-Y. 2012. clusterProfiler: an R package for comparing biological themes among gene clusters. Omics: a journal of integrative biology 16:284-287.

Zhuang DY, Jiang L, He QQ, Zhou P, and Yue T. 2015. Identification of hub subnetwork based on topological features of genes in breast cancer. Int $J$ Mol Med 35:664-674. 10.3892/ijmm.2014.2057

Zou K, Li Z, Zhang Y, Zhang H-y, Li B, Zhu W-I, Shi J-y, Jia Q, and Li Y-m. 2017. Advances in the study of berberine and its derivatives: a focus on anti-inflammatory and anti-tumor effects in the digestive system. Acta Pharmacologica Sinica 38:157-167.

552 
Figure 1

Process of the present study.

GEO: Gene Expression Omnibus; TCGA: The Cancer Genome Atlas; Overlapping DEGs:

Overlapping differentially expressed genes; GO: Gene Ontology; KEGG: Kyoto Encyclopedia of

Genes and Genomes; PPI: protein-protein interaction; TFs, transcription factors.

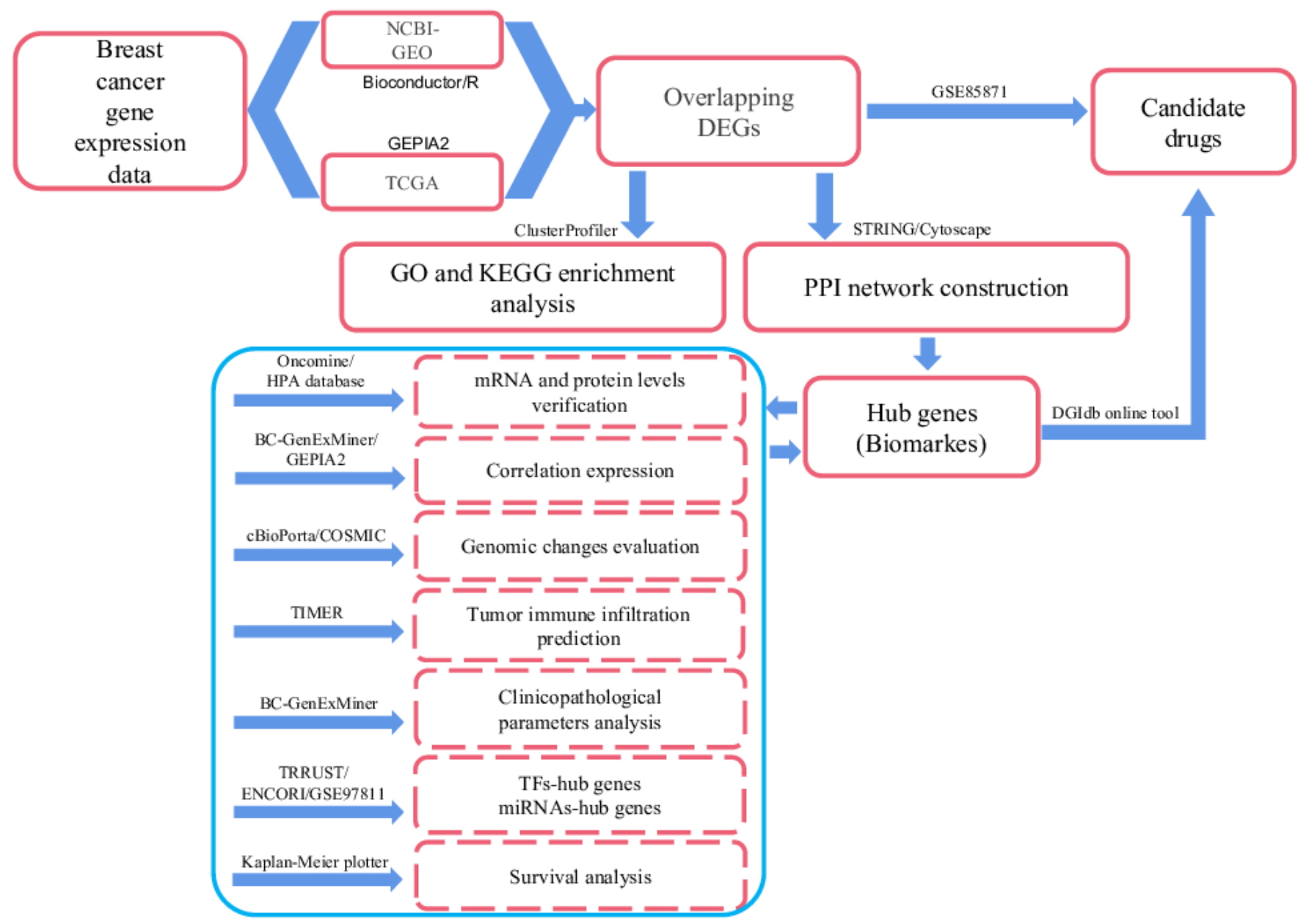




\section{Figure 2}

Identification of differentially expressed genes (DEGs) between breast cancer tumor tissues and normal tissues.

(A-E) Volcano plots of differential expression analysis for GSE3744 , GSE21422 , GSE42568 , GSE61304 , and GSE65194 . Red: up-regulated; Green: down-regulated. (F) Up-regulated DEGs selected by integrating 5 GEO databases. (G) Down-regulated DEGs selected by integrating 5 GEO databases. (H) Up-regulated overlapping DEGs obtained by integrating GEO datasets and TCGA BRCA dataset. (I) Down-regulated overlapping DEGs obtained by integrating GEO datasets and TCGA BRCA dataset. 

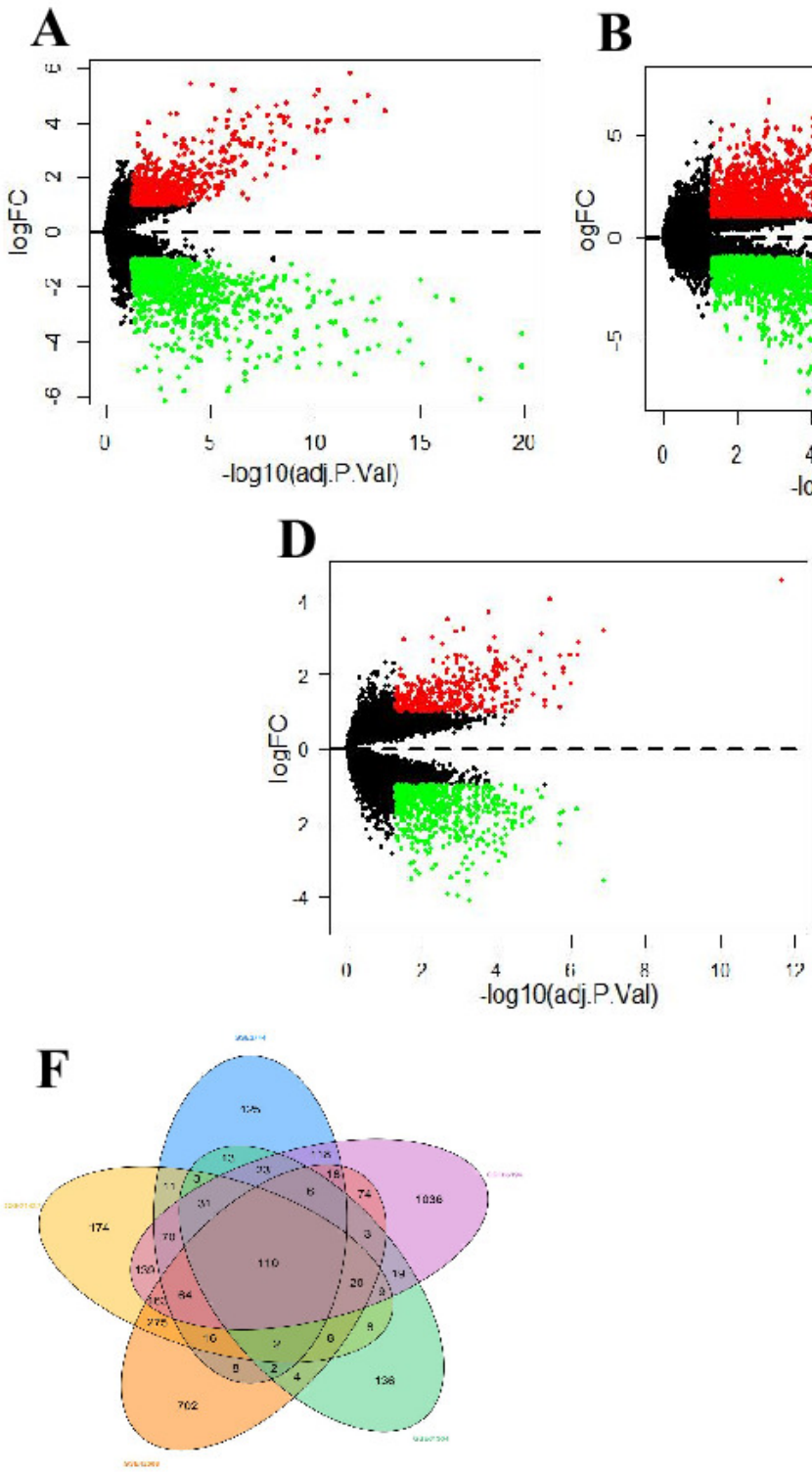
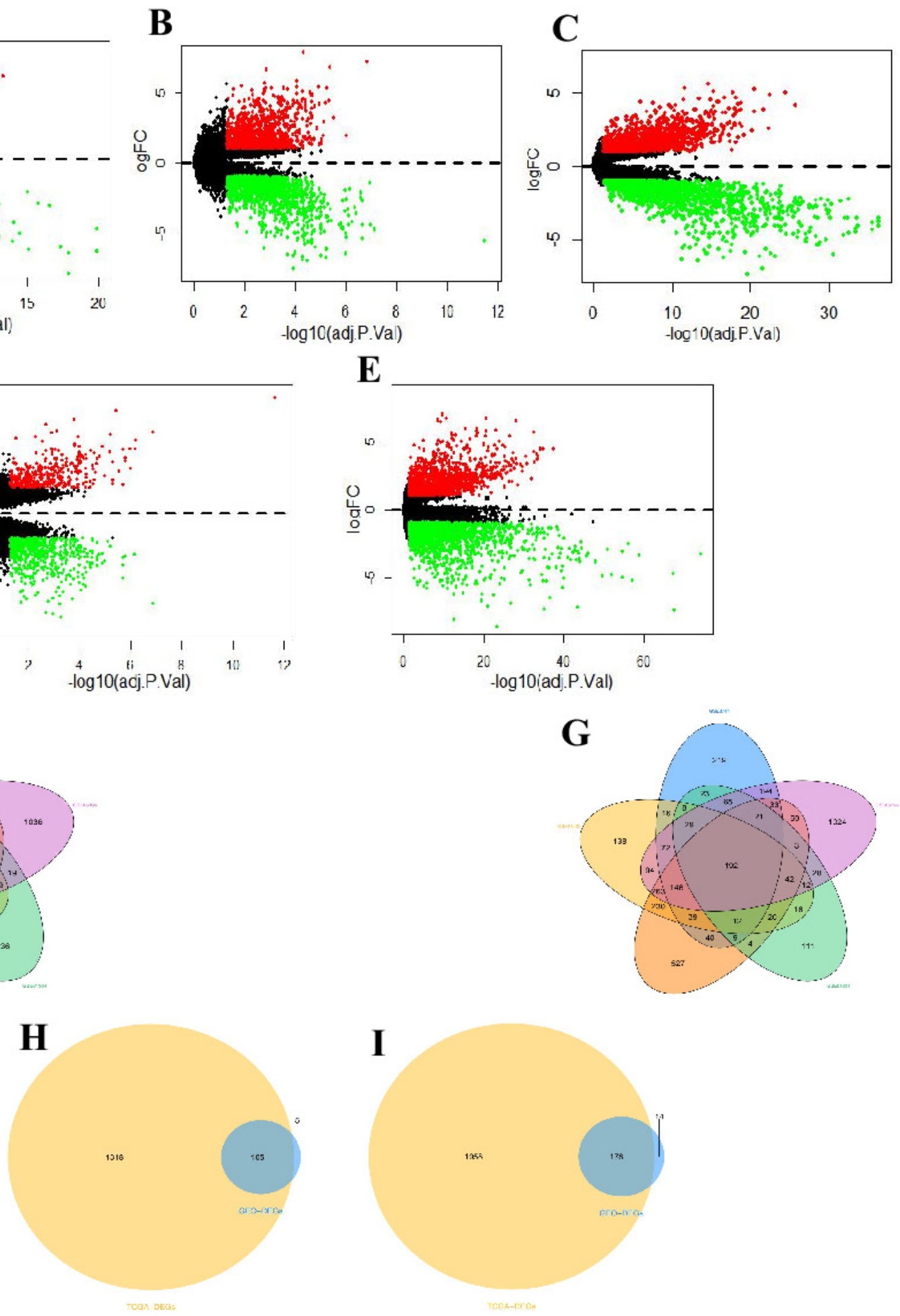


\section{Figure 3}

Expression verification of 6 hub genes.

(A) Hub genes expression in multiple types of human cancers from Oncomine database. Red: up-regulated; Blue: down-regulated. (B-K) Immunohistochemical staining analysis of hub genes in breast cancer based on the Human Protein Atlas. (B) Protein levels of RRM2 in normal tissue (staining: not detected; intensity: negative; quantity: none). (C) Protein levels of RRM2 in tumor tissue (staining: low; intensity: moderate; quantity: < 25\%). (D) Protein levels of CDC20 in normal tissue (staining: not detected; intensity: negative; quantity: none). (E) Protein levels of CDC20 in tumor tissue (staining: high; intensity: strong; quantity: 75\%-25\%). (F) Protein levels of CCNB2 in normal tissue (staining: not detected; intensity: negative; quantity: none). (G) Protein levels of CCNB2 in tumor tissue (staining: medium; intensity: moderate; quantity: 75\%-25\%). (H) Protein levels of CDK1 in normal tissue (staining: not detected; intensity: negative; quantity: none). (I) Protein levels of CDK1 in tumor tissue (staining: high; intensity: strong; quantity: 75\%-25\%). (J) Protein levels of CCNA2 in normal tissue (staining: not detected; intensity: negative; quantity: none). (K) Protein levels of CCNA2 in tumor tissue (staining: medium; intensity: strong; quantity: < $25 \%)$. There were no related immunohistochemical samples of BUB1B in the database. 
A

\begin{tabular}{|c|c|c|c|c|c|c|c|c|c|c|c|}
\hline \multirow{2}{*}{$\begin{array}{c}\text { Analysis Type by Cancer } \\
\text { Bladder Cancer }\end{array}$} & $\begin{array}{c}\text { Cancer } \\
\text { vs. } \\
\text { Normal } \\
\text { RRM2 }\end{array}$ & \multicolumn{2}{|c|}{$\begin{array}{l}\text { Cancer } \\
\text { vs. } \\
\text { Normal } \\
\text { CDC20 }\end{array}$} & \multicolumn{2}{|c|}{$\begin{array}{c}\text { Cancer } \\
\text { vs. } \\
\text { Normal } \\
\text { CCNB2 }\end{array}$} & \multicolumn{2}{|c|}{$\begin{array}{c}\text { Cancer } \\
\text { vs. } \\
\text { Normal } \\
\text { BUBıB }\end{array}$} & \multicolumn{2}{|c|}{$\begin{array}{l}\text { Cancer } \\
\text { vs. } \\
\text { Normal } \\
\text { CDK1 }\end{array}$} & \multicolumn{2}{|c|}{$\begin{array}{l}\text { Cancer } \\
\text { vs. } \\
\text { Normal } \\
\text { CCNA2 }\end{array}$} \\
\hline & 6 & 6 & & 4 & & 5 & & 6 & & 3 & \\
\hline Brain and CNS Cancer & 1 & 5 & & 6 & & 5 & 3 & 9 & 1 & 4 & 1 \\
\hline Breast Cancer & 161 & 19 & & 25 & & 19 & & 25 & 1 & 13 & 2 \\
\hline Cervical Cancer & 4 & 3 & & 3 & & 3 & & 3 & & 2 & \\
\hline Colorectal Cancer & 12 & 6 & & 7 & & 8 & & 13 & & 6 & \\
\hline Esophageal Cancer & 2 & 2 & & 2 & & 3 & & 3 & & 1 & \\
\hline Gastric Cancer & 3 & 2 & & 2 & & 2 & & 6 & & 3 & \\
\hline Head and Neck Cancer & 6 & 8 & & 5 & & 4 & & 7 & & 4 & \\
\hline Kidney Cancer & 2 & & & 2 & & & & 2 & 1 & & \\
\hline Leukemia & 7 & 1 & 5 & 3 & 7 & & 3 & 1 & 8 & 1 & 5 \\
\hline Liver Cancer & 4 & 3 & & 3 & & 4 & & 4 & & 4 & \\
\hline Lung Cancer & 13 & 12 & & 11 & & 15 & & 16 & & 13 & \\
\hline Lymphoma & \begin{tabular}{ll|}
13 & 1 \\
\end{tabular} & 8 & 1 & 3 & 1 & 3 & 1 & 6 & 1 & 3 & 1 \\
\hline Melanoma & 2 & 1 & & 1 & & 1 & 1 & 1 & & & \\
\hline Myeloma & 2 & & 2 & & & & & & 2 & & 1 \\
\hline Other Cancer & 6 & 5 & 2 & 5 & 5 & 6 & 2 & 7 & 3 & 5 & \\
\hline Ovarian Cancer & 4 & 4 & & 3 & & 4 & & 5 & & 2 & \\
\hline Pancreatic Cancer & 2 & 2 & & 2 & & 2 & 1 & 3 & & 1 & \\
\hline Prostate Cancer & 2 & & & 2 & & & & & & 1 & \\
\hline Sarcoma & 10 & 12 & 1 & 11 & 1 & 12 & & 12 & & 12 & \\
\hline Significant Unique Analyses & \begin{tabular}{|l|l|}
116 & 10
\end{tabular} & 97 & 11 & 98 & 14 & 95 & 11 & 128 & 17 & 77 & 10 \\
\hline Total Unique Analyses & 449 & 41 & & 415 & & 41 & & 455 & & 46 & 7 \\
\hline
\end{tabular}

B

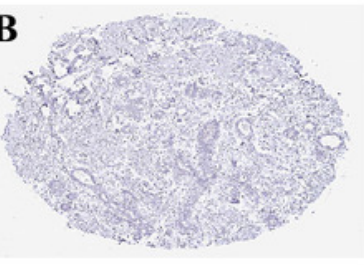

D

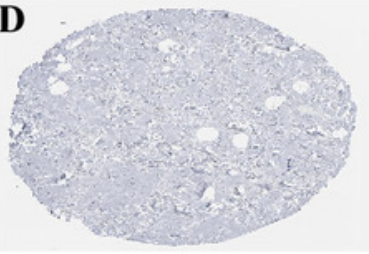

F

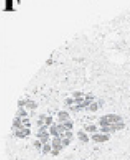

H

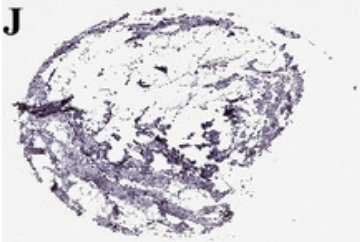

C

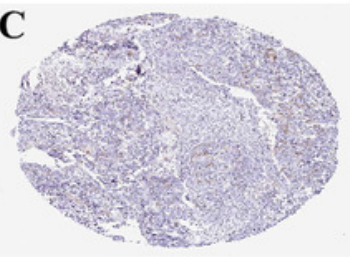

$\mathbf{E}$

G

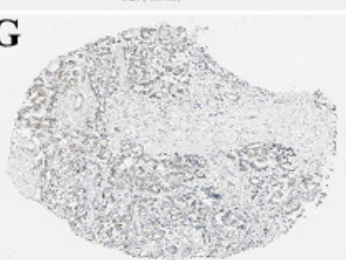

I

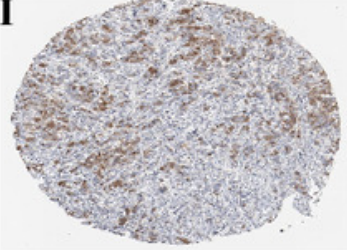

K

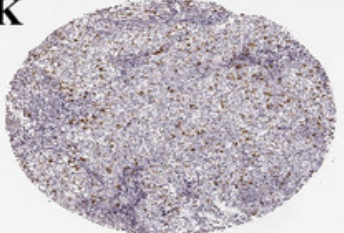


Figure 4

Gene Ontology (GO) and Kyoto Encyclopedia of Genes and Genomes (KEGG) pathway enrichment analysis of overlapping DEGs by ClusterProfiler and GOplot packages of R.

(A) GO analysis of up-regulated overlapping DEGs. (B) GO analysis of down-regulated

overlapping DEGs. BP, biological process. CC, cellular component. MF, molecular function. (C) Dot plot of KEGG pathway enrichment analysis. 

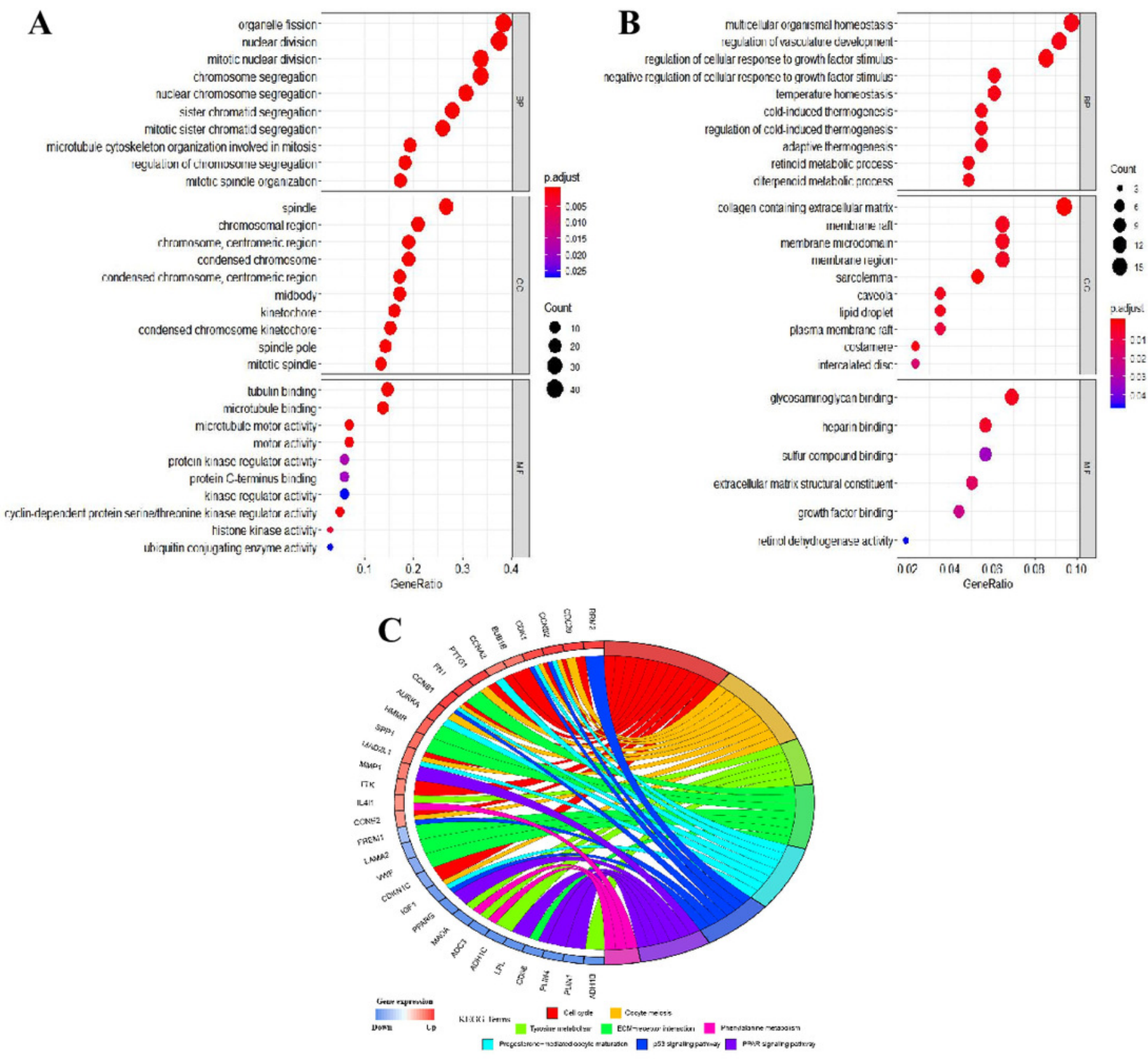


\section{Figure 5}

TFs-hub genes and miRNAs-hub genes regulatory networks.

(A) TFs-hub genes network obtained from TRRUST database. Red rhomboids: hub genes;

Ellipses: TFs; Red ellipses: up-regulated genes in overlapping DEGs; Blue ellipses: downregulated genes in overlapping DEGs; Delta-shaped arrows: activation of hub genes by TFs; Half circular arrows: repression of hub genes by TFs. TFs, transcription factors. (B) miRNAshub genes network. Red diamonds: hub genes; Red rectangles: up-regulated miRNAs; Blue rectangles: down-regulated miRNAs.
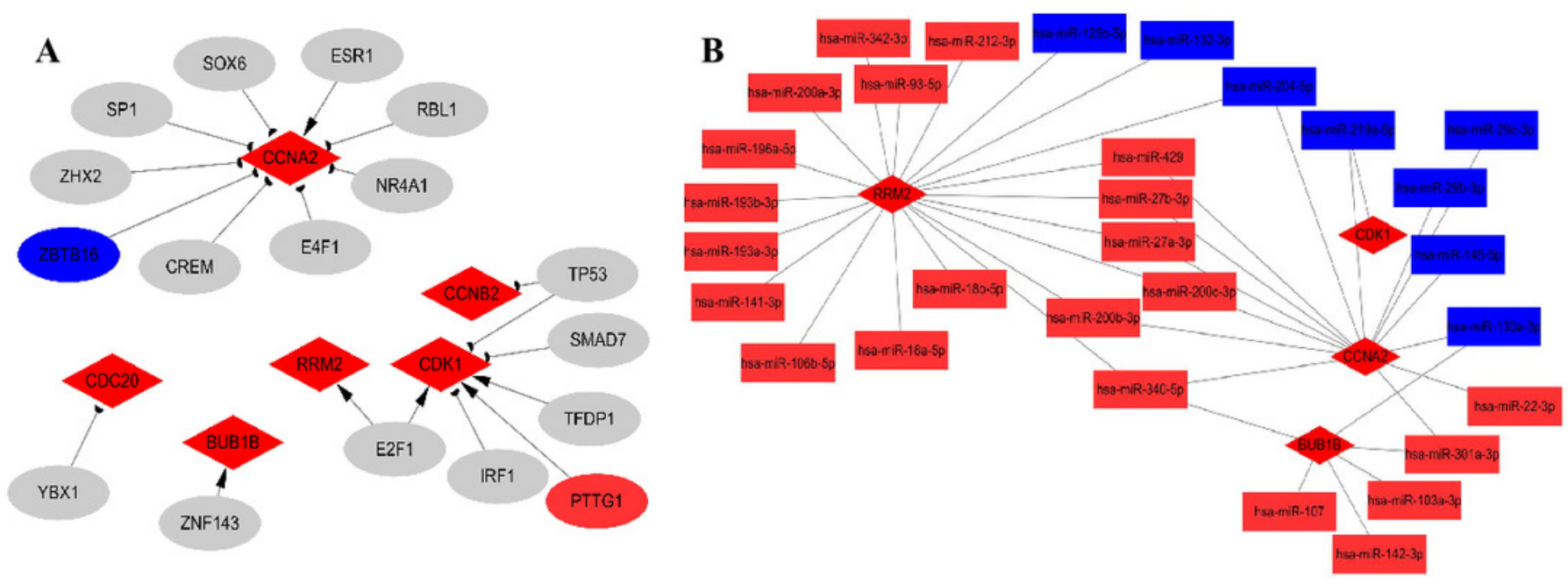


\section{Figure 6}

OS (A), RFS (B), and DMFS (C) analysis of RRM2, CDC20, CCNB2, BUB1B/SSK1, CDK1/CDC2, and CCNA2/CCNA in breast cancer based on Kaplan Meier-Plotter.

The patients were split into high and low expression groups according to the median expression of hub genes. OS, overall survival; RFS, relapse free survival; DMFS, distant metastasis-free survival.
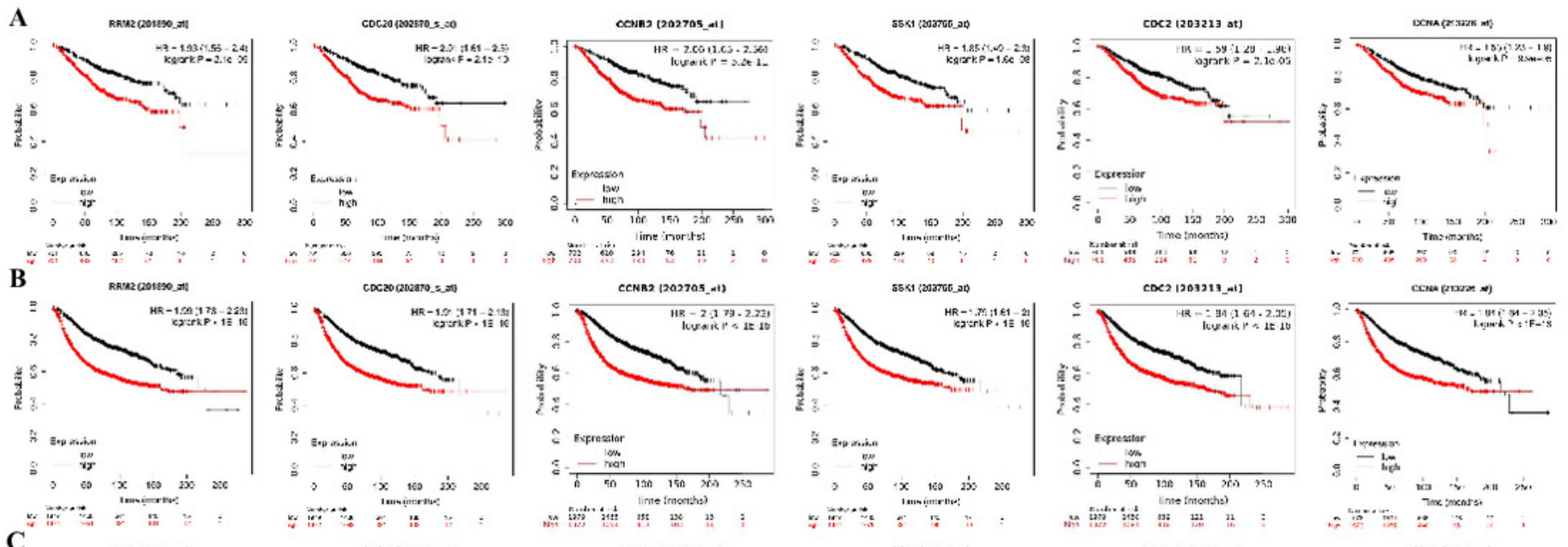

in srawe
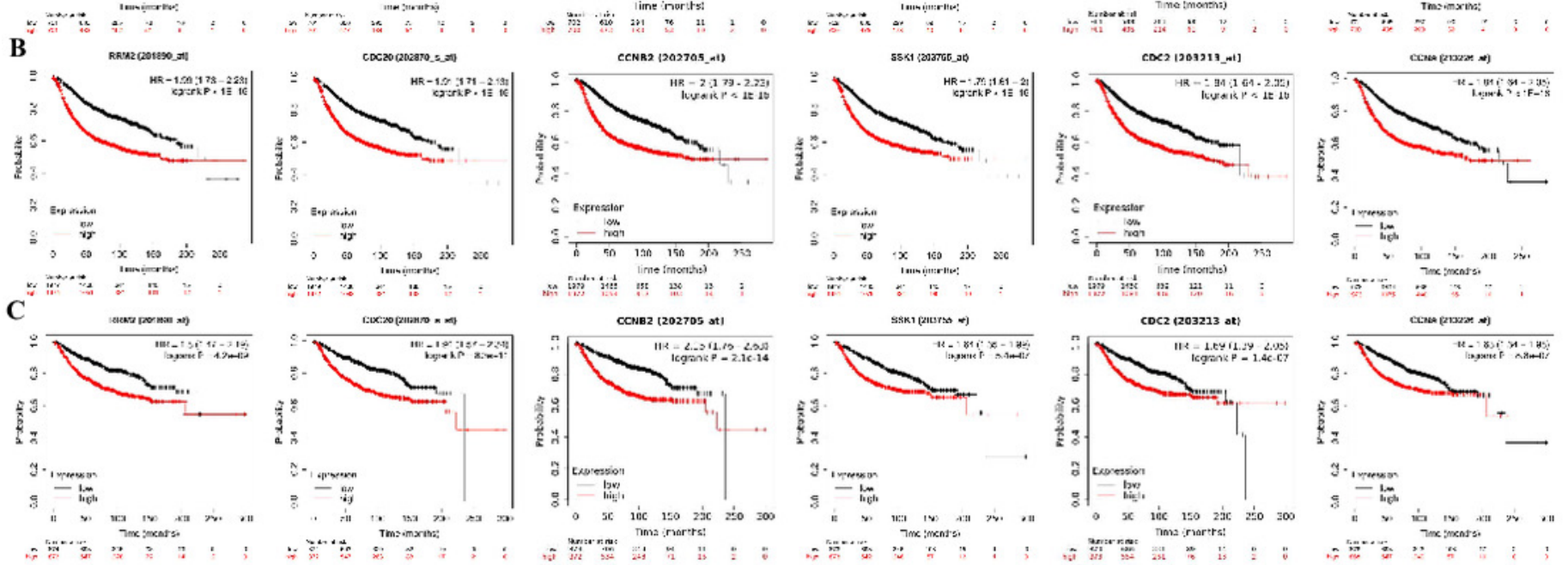
Figure 7

Traditional Chinese medicine (TCM) ingredients-overlapping DEGs-pathways networks.

(A) Japonicone A. (B) Nitidine chloride. (C) Berberine hydrochloride. (D) 1B-

hydroxyalantolactone. (E) Britanin. (F) Tanshinone IIA.Yellow diamond: TCM ingredient; Blue diamond: down-regulated gene; Red diamond: up-regulated gene; Green rectangle: KEGG pathway.

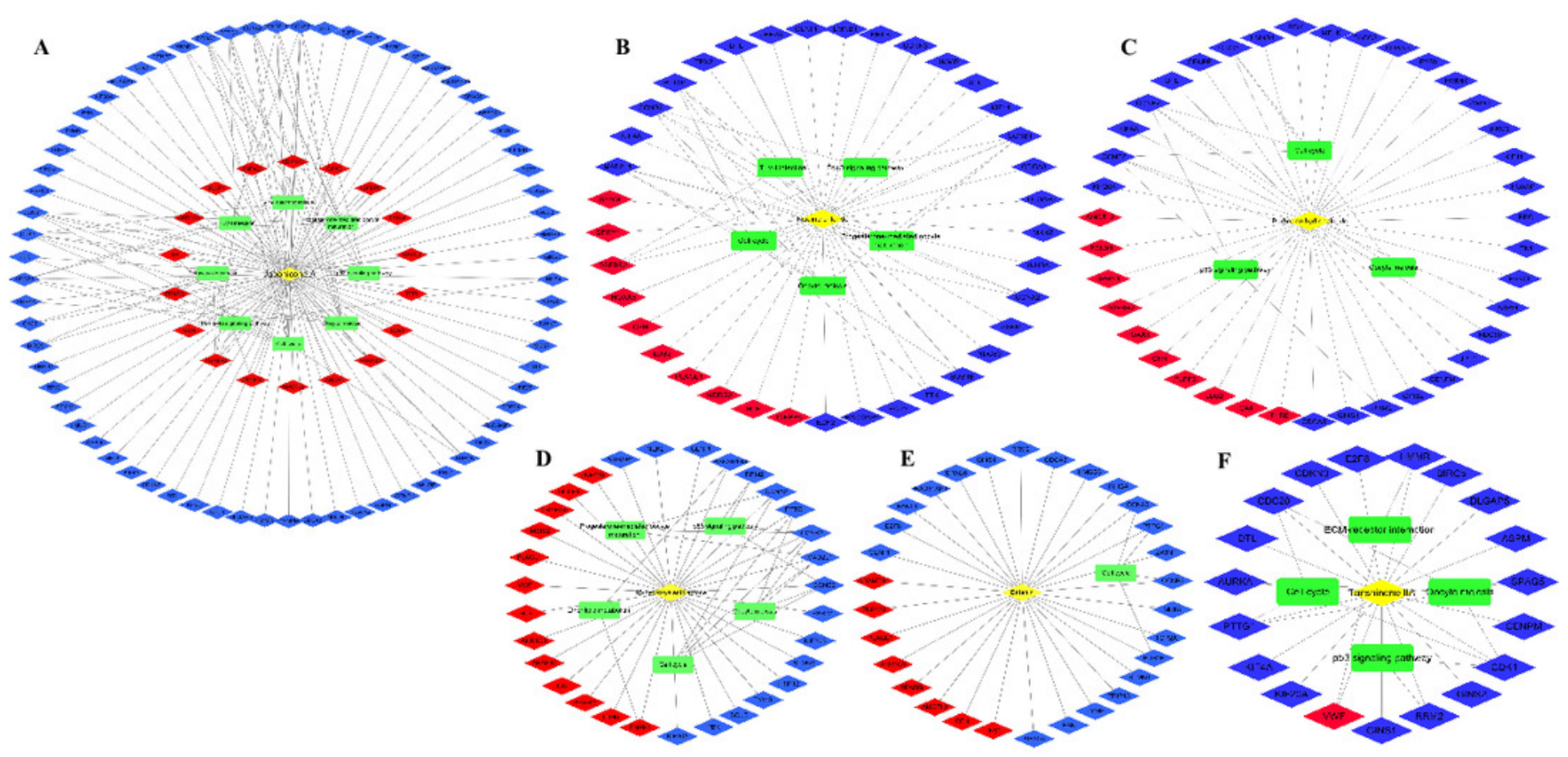




\section{Table 1 (on next page)}

Characteristics of datasets in this study. 


\begin{tabular}{ccc}
\hline Expression profile dataset & Breast cancer & Normal \\
\hline GSE3744 & 40 & 7 \\
GSE21422 & 14 & 5 \\
GSE42568 & 104 & 17 \\
GSE61304 & 58 & 4 \\
GSE65194 & 167 & 11 \\
TCGA BRCA & 1085 & 112 \\
\hline
\end{tabular}

1 
Table 2 (on next page)

Antineoplastic drugs targeting hub genes based on DGIdb database. 


\begin{tabular}{|c|c|c|c|c|c|}
\hline Target & Drug & Type & Sources & PMIDs & Score \\
\hline \multirow{15}{*}{ RRM2 } & \multirow{5}{*}{ CLADRIBINE } & \multirow{5}{*}{ inhibitor } & \multirow{5}{*}{ DrugBank } & 17852710 & \multirow{5}{*}{6} \\
\hline & & & & 16316309 & \\
\hline & & & & 19576186 & \\
\hline & & & & 9923554 & \\
\hline & & & & 19715446 & \\
\hline & \multirow{3}{*}{$\begin{array}{l}\text { GALLIUM } \\
\text { NITRATE }\end{array}$} & \multirow{3}{*}{ inhibitor } & \multirow{3}{*}{ ChemblInteractions DrugBank } & 12776257 & \multirow{3}{*}{5} \\
\hline & & & & 1335254 & \\
\hline & & & & 15651176 & \\
\hline & $\begin{array}{l}\text { MOTEXAFIN } \\
\text { GADOLINIUM }\end{array}$ & inhibitor & TdgClinicalTrial DrugBank TTD & - & 3 \\
\hline & HYDROXYUREA & inhibitor & $\begin{array}{c}\text { GuideToPharmacologyInteractions } \\
\text { ChemblInteractions }\end{array}$ & - & 2 \\
\hline & CLOFARABINE & inhibitor & $\begin{array}{c}\text { GuideToPharmacologyInteractions } \\
\text { ChemblInteractions }\end{array}$ & - & 2 \\
\hline & GEMCITABINE & inhibitor & $\begin{array}{l}\text { ClearityFoundationClinicalTrial } \\
\text { GuideToPharmacologyInteractions }\end{array}$ & - & 2 \\
\hline & $\begin{array}{l}\text { FLUDARABINE } \\
\text { PHOSPHATE }\end{array}$ & inhibitor & ChemblInteractions & - & 1 \\
\hline & TRIAPINE & - & TdgClinicalTrial & - & 1 \\
\hline & FLUDARABINE & inhibitor & GuideToPharmacologyInteractions & - & 1 \\
\hline \multirow{6}{*}{ CDK1 } & \multirow{5}{*}{ DINACICLIB } & \multirow{5}{*}{ inhibitor } & MyCancerGenome & \multirow{5}{*}{-} & \multirow{5}{*}{6} \\
\hline & & & ClearityFoundationClinicalTrial & & \\
\hline & & & GuideToPharmacologyInteractions & & \\
\hline & & & ChemblInteractions CancerCommons & & \\
\hline & & & MyCancerGenomeClinicalTrial & & \\
\hline & ALVOCIDIB & inhibitor & $\begin{array}{c}\text { MyCancerGenome TdgClinicalTrial } \\
\text { ChemblInteractions DrugBank }\end{array}$ & 11752352 & 5 \\
\hline
\end{tabular}




\begin{tabular}{|c|c|c|c|c|c|}
\hline & Roniciclib & inhibitor & $\begin{array}{l}\text { GuideToPharmacologyInteractions } \\
\text { ChemblInteractions CancerCommons }\end{array}$ & - & 3 \\
\hline & AT-7519 & inhibitor & $\begin{array}{c}\text { GuideToPharmacologyInteractions } \\
\text { ChemblInteractions DrugBank }\end{array}$ & - & 3 \\
\hline & AZD-5438 & inhibitor & $\begin{array}{c}\text { GuideToPharmacologyInteractions } \\
\text { ChemblInteractions }\end{array}$ & - & 2 \\
\hline & TG-02 & inhibitor & $\begin{array}{c}\text { GuideToPharmacologyInteractions } \\
\text { ChemblInteractions }\end{array}$ & - & 2 \\
\hline & CHEMBL1236539 & inhibitor & GuideToPharmacologyInteractions & - & 1 \\
\hline & RG-547 & inhibitor & ChemblInteractions & - & 1 \\
\hline & SELICICLIB & inhibitor & ChemblInteractions & - & 1 \\
\hline \multirow{3}{*}{ CCNA2 } & SURAMIN & - & $\mathrm{NCI}$ & 10208280 & 2 \\
\hline & CORDYCEPIN & - & $\mathrm{NCI}$ & 11566717 & 2 \\
\hline & GENISTEIN & - & NCI & 9664138 & 2 \\
\hline
\end{tabular}

1 